\title{
Chirurgische Behandlung von Skelettmetastasen
}

\author{
Thomas Gösling, Ulf Brunnemer, Christian Krettek
}

\section{Zusammenfassung}

Die Metastasierung stellt eine Generalisierung eines Tumorleidens im Körper dar. Die Behandlung von Skelettmetastasen ist daher interdisziplinär vorzunehmen und erfordert ein stadien- und patientenadaptiertes Vorgehen. Bei unbekanntem Primarius oder langem Intervall zwischen Tumor und Auftreten einer Metastasierung muss auch an das Vorliegen eines primären Knochentumors bzw. eines Zweitkarzinoms gedacht werden. Bei Zweifel ist hier dringend eine Biopsie zu empfehlen. An chirurgischen Therapiemöglichkeiten stehen die alleinige Stabilisierung, die Stabilisierung mit Radiatio, die intraläsionale Resektion mit Radiatio sowie die weite bzw. marginale Resektion zur Verfügung. Verschiedene Rekonstruktionsverfahren bieten Vor- und Nachteile. Die Therapieentscheidung muss der aktuellen tienten Rechnung tragen. Der onkologische Fortschritt mit deutlicher Verbesserung der Lebenserwartung führt zu einer deutlich aggressiveren Herangehensweise in der chirurgischen Lokaltherapie. und zu erwartenden Situation des Pa-

\section{Surgical Treatment of Skeletal Metastases}

Metastasis constitutes a generalisation of tumour disease within the body. The treatment of skeletal metastases must therefore be an interdisciplinary process and demands a procedure that is appropriate for the stage of disease and for the patient. In cases of an unknown primary lesion or a long interval between detection of the tumour and the occurrence of metastases, the presence of a primary bone tumour or, respectively, a secondary tumour must be taken into consideration. In cases of doubt a biopsy is urgently recommended. The options available for surgical treatment include stabilisation alone, stabilisation with radiotherapy, interlesional resection with radiotherapy, and wide or, alternatively, marginal resection. Various reconstruction procedures have their advantages and disadvantages. The therapeutic decision-making must take the current and the to be expected future condition of the patient into consideration. Advances in oncology with decisive improvements in life expectancy have led to markedly more aggressive approaches in local surgical therapy.

Metastasen werden auch als sekundäre Neubildungen bezeichnet.

Knochenmetastasen sind Fernabsiedlungen eines primären Tumors im Knochen. Die Metastasierung zeigt eine Generalisierung der Erkrankung an. In der Regel handelt es sich bei den primären Tumoren um Karzinome. Andere Tumorarten (z.B. Sarkome) sind jedoch auch möglich.

OP-JOURNAL 2010; 26: 202-206

(c) Georg Thieme Verlag KG Stuttgart · New York DOI http://dx.doi.org/10.1055/s-0030-1250613
Der Knochen ist neben der Lunge und der Leber die häufigste Lokalisation von Metastasen. Die Verteilung unterscheidet sich jedoch zwischen den einzelnen Primärtumorarten. Die häufigsten Tumoren mit Knochenmetastasen sind die Karzinome von Mamma, Prostata, Lunge, Niere und Schilddrüse $[3,4]$.
Galten Metastasen vor einigen Jahren noch als sicherer Prädiktor eines frühen bis mittelfristigen Ablebens, können durch Fortschritte der Onkologie Patienten mit Knochenmetastasen durchaus bei hoher Lebensqualität eine mehrjährige Lebenserwartung haben. Unter gewissen Umständen kann man fast schon von einer „chronischen Erkrankung“ sprechen. Dies ist jedoch entschieden von der Art des Primärtumors und dem Ansprechen der adjuvanten Therapie abhängig. Des Weiteren haben das Intervall zwischen Diagnose des Primärtumors und dem Auftreten von Metastasen, die lokale Verteilung der Metastasen und die Anzahl der Metastasen einen Einfluss auf die Prognose.

Die Behandlung von Knochenmetastasen sollte daher immer interdisziplinär erfolgen. Wünschenswert ist eine Besprechung im Rahmen von Konferenzen.

Knochenmetastasen entstehen in der Regel durch hämatogene Aussaat. Die Veränderungen im Knochen werden überwiegend durch die Fähigkeit der Tumorzellen hervorgerufen, Osteoblasten und/oder Osteoklasten zu stimulieren. Hierdurch entstehen osteoblastische bzw. osteolytische Metastasen, wobei beide Formen auch parallel vorkommen können. Bisphosphonate greifen hier an und sind ein wichtiger Faktor in der Behandlung von Skelettmetastasen.

Zwar existieren von der Deutschen Gesellschaft für Orthopädie und Orthopädische Chirurgie (DGOOC) Leitlinien zur Behandlung von Knochenmetastasen, diese sind jedoch weniger präzise als die für die Behandlung primärer maligner Knochentumoren. Dies liegt an der Komplexität der Gesamterkrankung, in der neben der Tumorentität v.a. der Lokalbefund, der Allgemeinzustand des Patienten und die zu erwartende Überlebenszeit $\mathrm{zu}$ berücksichtigen sind. Die Behandlung ist individuell $\mathrm{zu}$ planen und bedarf daher einiger Erfahrung. In 
Tab. 1 Frakturrisiko metastasenbefallener Röhrenknochen nach Mirels.

\begin{tabular}{llll}
\multicolumn{5}{c}{ Score } & & \\
\hline Faktor & 1 & 2 & 3 \\
\hline Lokalisation & obere Extremität & untere Extremität & peritrochantär \\
\hline Schmerz & gering & mäßig & schwer \\
\hline Läsion & osteoblastisch & gemischt & osteolytisch \\
\hline Größe & $<1 / 3$ der kortikalen & $1 / 3-2 / 3$ der kortikalen & $>2 / 3$ der kortikalen \\
& Zirkumferenz & Zirkumferenz & Zirkumferenz
\end{tabular}

Score $\geq 9$ Punkte: hohes Frakturrisiko, OP-Indikation gegeben!

Tab. 2 Score nach Bauer: Prognose von Patienten mit Knochenmetastasen.

\begin{tabular}{ll} 
Charakteristik & Punktwert \\
\hline Fehlen von viszeralen Metastasen & 1 \\
\hline Fehlen einer pathologischen Fraktur & 1 \\
\hline 1 solitäre Skelettmetastase & 1 \\
\hline Primärtumor ist nicht Lunge & 1 \\
\hline Primärtumor ist Mamma, Niere, Lymphom oder Myelom & 1 \\
\hline Prognose & \\
\hline 4-5 Punkte & 1-Jahres-Überlebensrate von 50\% \\
\hline 2-3 Punkte & 1-Jahres-Überlebensrate von 25\% \\
\hline 0-1 Punkte & Prognose <6 Monate
\end{tabular}

diesem Beitrag werden die wichtigsten chirurgischen Prinzipien in der Behandlung von Knochenmetastasen beschrieben. Neben der chirurgischen und medikamentösen Therapie besitzt die Strahlentherapie als alleinige oder adjuvante Therapie eine große Bedeutung in der Behandlung der Skelettmetastasen. Bei einigen Tumoren (speziell Mamma und Prostata) kann die Radiation sogar zu einer Remineralisierung von fast 60\% der Läsion und somit zum Stabilitätsgewinn führen. Auf die nicht chirurgischen Therapien wird in diesem Beitrag nicht im Detail eingegangen.

\section{Diagnostik}

Skelettmetastasen können aus unterschiedlichen Gründen evident werden. In der Regel werden die Patienten bei bekanntem Tumorleiden durch ihren onkologisch betreuenden Arzt dem Chirurgen/Orthopäden zugewiesen. Skelettmetastasen werden hier bei Staginguntersuchungen im Rahmen der Primärdiagnostik oder Nachsorge häufig als asymptomatischer Zufallsbefund entdeckt. Eine beobachtete B-Symptomatik ist im Allgemeinen Ausdruck eines Tumorprogresses und sollte an das Vor-
Bei dem Verdacht einer Knochenmetastase sollte zur weiteren Therapieentscheidung ein Staging vorliegen bzw. veranlasst werden. Als minimale Anforderung sollte diese eine Skelettszintigrafie und eine Computertomografie des Thorax und des Abdomens beinhalten. Die Ganzkörper-MRT stellt eine Alternative zur Skelettszintigrafie dar.

Die lokale Computertomografie ist v.a. bei Metastasen im Bereich der Wirbelsäule und des Beckens sinnvoll. Im Bereich der Röhrenknochen kann die Computertomografie eine sinnvolle Ergänzung zu den konventionellen Röntgenaufnahmen darstellen, wenn eine präzisere Einschätzung der Stabilität eines metastatisch befallenen Knochens notwendig wird. Zur Einschätzung der Stabilität von Röhrenknochen ist international der Score nach Mirels anerkannt (Tab. 1).

Die lokale MRT ist zur Beurteilung der Stabilität der CT und dem konventionellen Röntgenbild unterlegen. Bedeutung besitzt die MRT v.a. bei geplanten weiten Resektionen, da sie am besten eine evtl. Weichteilkomponente und deren Lagebeziehung abbilden kann.

Eine Biopsie kann aus folgenden Gründen notwendig sein:

- unbekanntes Tumorleiden mit symptomatischer Knochenveränderung (primären Knochentumor ausschließen, Abb. 1)

- isolierte Metastase (Zweittumor ausschließen). Dies trifft umso mehr zu, je länger das Intervall zwischen bekanntem Tumor und vermuteter Metastase ist. tastasen vor, können jedoch aufgrund der minderen Knochenqualität auch bei osteoblastischen Metastasen beobachtet werden. Nicht selten findet sich die Situation, dass Patienten ohne bekanntes Tumorleiden mit einer metastasensuspekten Raumforderung vorgestellt werden. Hier ist ein besonderes Vorgehen durchzuführen (s.u.).

Das konventionelle Röntgenbild hat auch in der Diagnostik der Skelettmetastasen seinen uneingeschränkten Stellenwert.

Osteolytische Metastasen zeigen einen unscharfen Rand ohne Anzeichen einer Matrix oder reaktiven Knochenbildung. Im Bereich der langen Röhrenknochen muss vor Einleitung einer chirurgischen Therapie eine Mitabbildung der angrenzenden Gelenke erfolgen. 
Prinzipiell stehen aus chirurgischer Sicht folgende Therapieverfahren zur Verfügung: reine Stabilisierung, Stabilisierung mit adjuvanter Radiatio, Stabilisierung mit intraläsionaler Resektion und Radiatio, weite Resektion.

\section{Reine Stabilisierung}

Die reine Stabilisierung ohne Tumorresektion, und Radiatio führt zu keiner lokalen Tumorkontrolle. Sie ist im weit fortgeschrittenen Tumorstadium indiziert, um Patienten eine schnelle Schmerzlinderung zu bringen und eine Mobilisierung zu ermöglichen. Als Stabilisierungverfahren kann hier durchaus die Marknagelung mit Tumorverschleppung innerhalb des Kompartiments Knochen erfolgen.

\section{Reine Stabilisierung und Radiatio}

Die reine Stabilisierung ohne Tumorresektion, aber mit anschließender Radiatio führt zu einer kurzfristigen Tumorkontrolle. Aus diesem Grunde ist sie Patienten mit einer schlechten Prognose bei fortgeschrittenem Krankheitsverlauf vorbehalten. Ein klassisches Beispiel ist das multipel metastasierte Bronchialkarzinom mit schlechtem Ansprechen auf die Chemotherapie (Abb. 1). Bei Patienten, die ein Stable Disease unter Therapie aufweisen, sollte die Nagelung ohne chirurgische Tumorresektion aus unserer Sicht nicht erfolgen, da trotz nachfolgender Radiatio ein lokales Tumorrezidiv möglich ist und die Nagelung zu einer Verbreitung des Tumors führt (Abb. 2).

\section{Intraläsionale Resektion und Radiatio}

Dieses Verfahren ist wohl am weitesten verbreitet und am bekanntesten in der Behandlung von Skelettmetastasen und pathologischen Frakturen. Anders als bei einer weiten Resektion wird der Tumor in der Regel kürettiert, sodass die Resektion als intraläsional zu bezeichnen ist (s. Artikel Allgemeine Grundlagen in diesem Heft). Die Resektion gewährleistet keine langfristige Tumorkontrolle. Durch die Kombination mit der Radiatio kann bei strahlensensiblen Tumoren jedoch eine langfristige und bei weniger strahlensensiblen Tumoren eine mittelfristige lokale Tumorkontrolle erreicht werden. Der Vorteil der intraläsionalen gegenüber der weiten Resektion besteht v.a. in der leichteren OP-Technik und der geringeren Morbidität im Bereich des Knochens, wodurch häufig eine Rekonstruktion ohne Einsatz von Tumorpro-

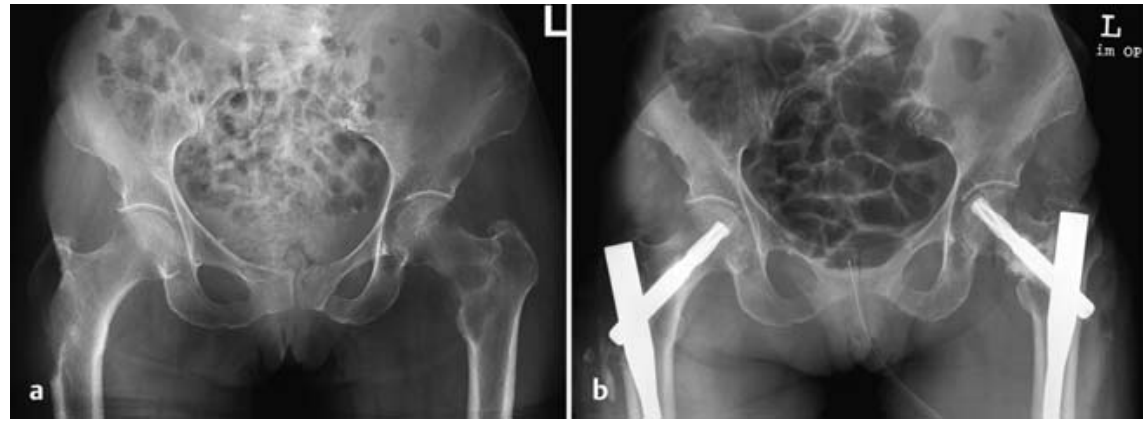

Abb. $\mathbf{1}$ a und $\mathbf{b}$ a Das Röntgenbild gehört zu einer Patientin, die erstmalig mit Schmerzen im Bereich beider Hüften klinisch auffällig wurde. Das konventionelle Röntgenbild zeigte beidseits Osteolysen mit dem Verdacht auf Metastasen. Die Stanzbiospie ergab den Befund eines nicht kleinzelligen Bronchialkarzinoms. $\mathbf{b}$ Bei fortgeschrittenem Lokalbefund in der Lunge und diffuser viszeraler und ossärer Metastasierung erfolgte die Entscheidung zur reinen Stabilisierung mittels intramedullärem Kraftträger und postoperativer Radiatio. Die Patientin konnte umgehend mobilisiert werden, verstarb jedoch 8 Monate nach Diagnosestellung.
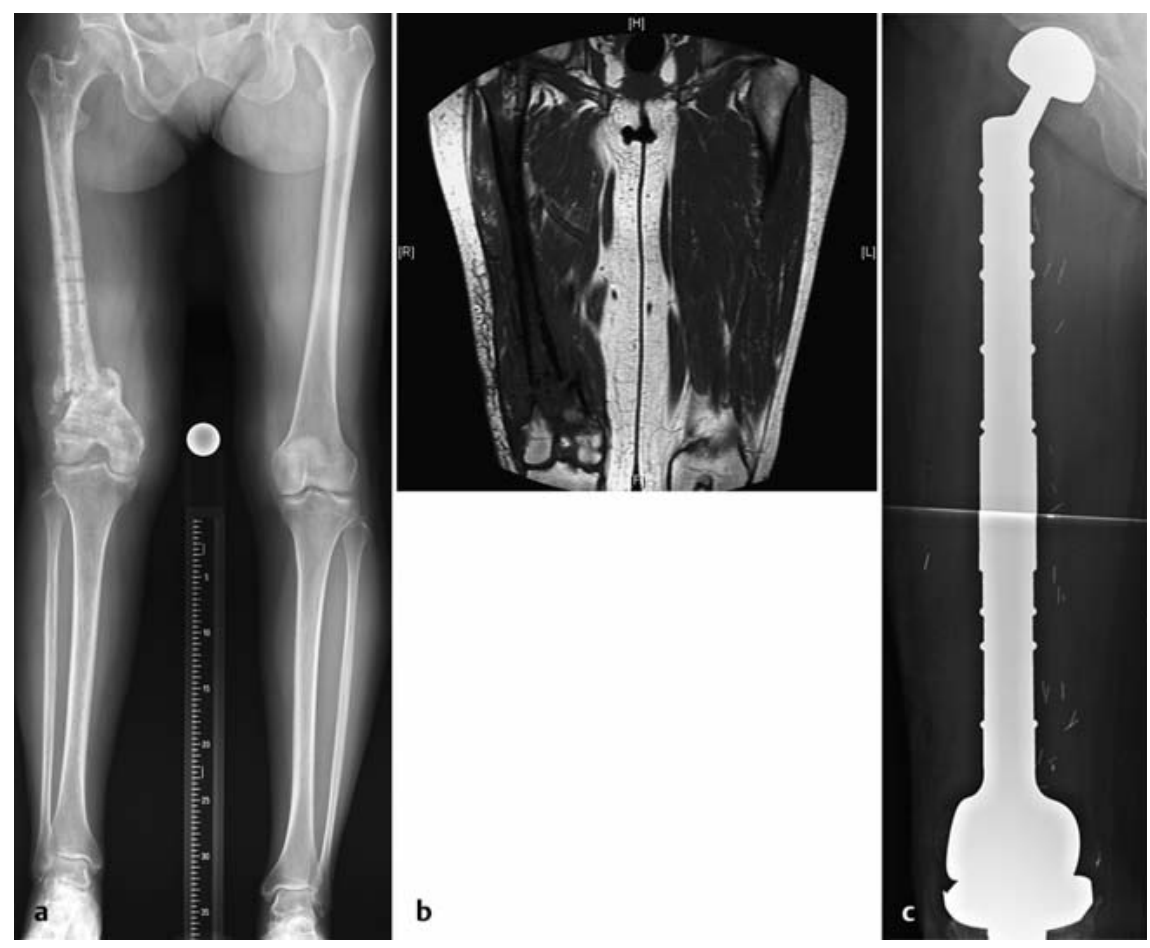

Abb. 2 a bis c a 42-jährige Patientin, die vor 8 Jahren wegen Mammakarzinoms operiert wurde. Fünf Jahre später Darstellung einer isolierten Metastase des Femurs, welche mittels Nagelosteosynthese und postoperativer Radiatio behandelt wurde. Bei ausbleibender Heilung und Implantatversagen erneuter Verfahrenswechsel. Die Patientin wurde dann mit Pseudarthrose, Beinverkürzung und Mobilisierung an Unterarmgehstützen vorgestellt. b Im MRT stellte sich der Verdacht einer Markraummetastasierung rechts. Bei einer Biopsie im Bereich der Pseudarthrose und im Bereich des proximalen Femurs konnte histologisch das Vorliegen einer Metastase des vordiagnostizierten Mammakarzinoms nachgewiesen werden. c Bei isoliertem Nachweis einer Metastasierung im Femur und sehr gutem Allgemeinzustand erfolgte die Entscheidung zur weiten totalen Femurresektion mit anschließendem prothetischem Ersatz.

thesen möglich ist. Zu beachten ist, dass einige Tumoren (z.B. Nierenzellkarzinom, Schilddrüsenkarzinom) reich vaskularisiert sind und bei der intraläsionalen Resektion erheblich bluten. Speziell im Becken- und Wirbelsäulenbereich, aber auch bei großen Tumoren an den Extremitäten sollte eine vorherige Em- bolisierung der Läsion in Erwägung gezogen werden. Der verbliebene Defekt muss je nach Größe und Belastung der Extremität gefüllt werden. In der Regel erfolgt dies alloplastisch mit Knochenzement im Sinne einer Verbundosteosynthese (Abb. 3). Der Zement hat verschiedene Effekte. Er führt durch Hitze- 


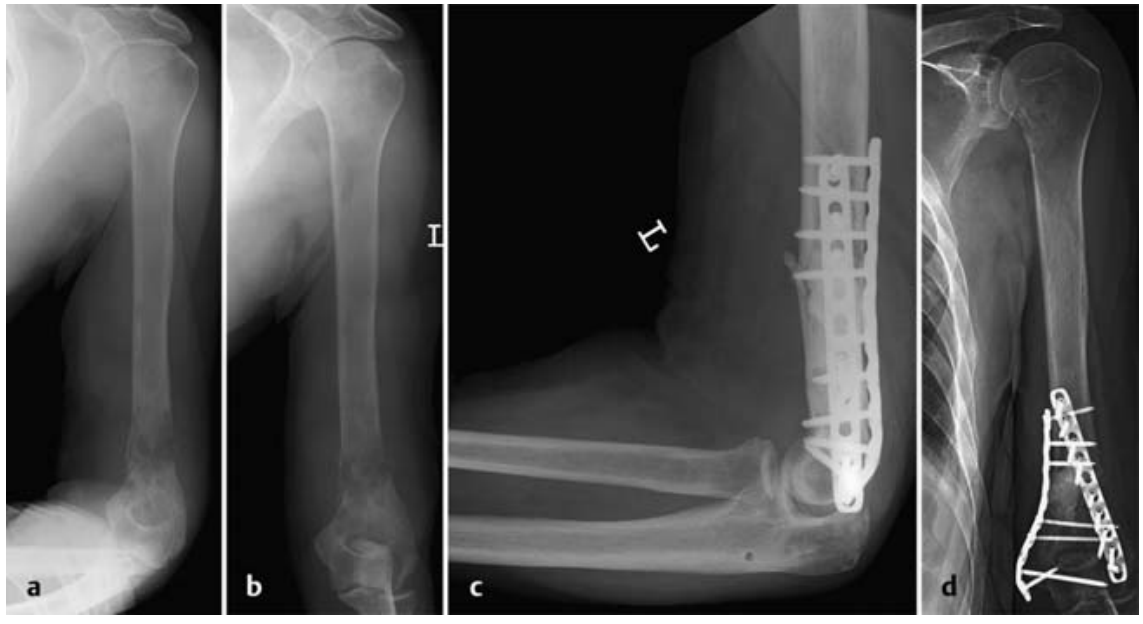

Abb. 3 a bis d a,b 62-jähriger Patient mit pathologischer suprakondylärer Humerusfraktur bei bekanntem Bronchialkarzinom. c, d Die Behandlung erfolgte mit intraläsionaler Resektion und Verbundosteosynthese.

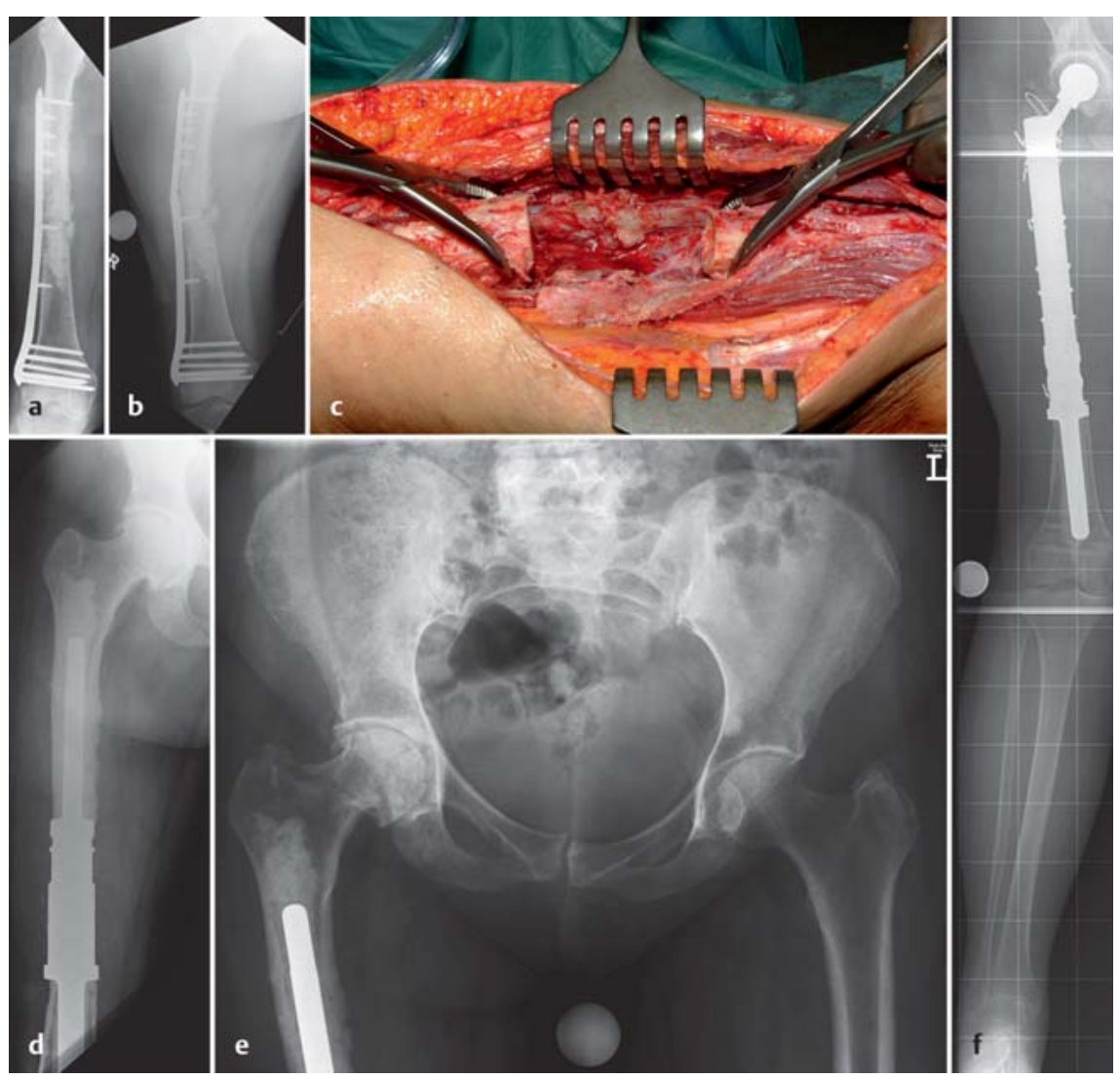

Abb.4a bis $\mathbf{f}$ Patientin, die als 51-Jährige an einem Mammakarzinom erkrankt ist. a 11 Jahre später erfolgte bei pathologischer Fraktur die auswärtige Verbundosteosynthese. b 14 Monate später wurde die Patientin bei Plattenbruch zugewiesen. c Intraoperatives Bild der marginalen Resektion. d Implantation eines Diaphysenersatzes. e Zwei Jahre später Auftreten einer Metastase mit Fraktur im Bereich des Schenkelhalses. f Umwandlung des Diaphysenersatzes in einen proximalen Femurersatz. Die Patientin erlitt 2 Jahre später eine erneute Fraktur (nun linksseitiger Oberschenkel, keine Abbildung).

entwicklung zu einer Erweiterung des intraläsionalen Resektionsrands (s. Artikel Allgemeine Grundlagen) und zu einer gewissen Blutstillung. Des Weiteren ist die Verbundosteosynthese sofort voll be- lastbar. Zu einer Heilung des Knochens und somit einem langfristig belastbaren Implantat kann es jedoch nur kommen, wenn um den Zement herum eine Knochenschale verbleibt, die eine knöcherne
Überbrückung gewährleistet (Abb.4). Eine spezielle Situation ergibt sich am Hüftgelenk. Durch die biomechanischen Gegebenheiten mit starker exzentrischer Belastung versagt die Verbundosteosynthese bei größeren Defekten oftmals sehr früh, sodass hier auch bei intraläsionaler Resektion der endoprothetische Ersatz erfolgen sollte. Viele Probleme lassen sich mit einer zementierten Standardprothese lösen [2]. Nur in wenigen Fällen muss bei intraläsionaler Resektion auf eine Tumorprothese zurückgegriffen werden.

\section{Weite Resektion}

Die weite Resektion bietet die höchste Wahrscheinlichkeit einer langfristigen lokalen Tumorkontrolle. Speziell bei Patienten mit isolierter Knochenmetastase, fehlenden viszeralen Metastasen und/ oder einem sehr guten Ansprechen der onkologischen Therapie mit Remission sollte die weite Resektion in Erwägung gezogen werden. Hinzu kommen unter speziellen Gegebenheiten wenig strahlensensible Metastasen. Die Resektion und Rekonstruktionen erfolgen ähnlich der der primären Knochentumorchirurgie. Auch im Bereich der Wirbelsäule (s. Artikel Chirurgische Therapieoptionen primärer und sekundärer Tumoren der Wirbelsäule in diesem Heft) und des Beckens (Abb.5) haben sich Indikationen zur weiten Resektion von Metastasen ergeben. Speziell bei Tumorerkrankungen mit langfristig guter Prognose müssen auch biologische Verfahren in Erwägung gezogen werden (Abb. 6). Gemäß der lokalen Konfiguration ist gelegentlich lediglich eine marginale Resektion möglich. Es bleibt jedoch festzuhalten, dass heute die weite Resektion von Knochenmetastasen eher die Ausnahme darstellt und die Indikation in enger Absprache zwischen Onkologen, Chirurgen und Patienten gestellt werden sollte. Die Metastasierung stellt eine Generalisierung der Erkrankung dar. Zukünftige Verbesserungen in der onkologischen Therapie spezieller Erkrankungen werden jedoch sicherlich zur Ausweitung der Indikation zur weiten Resektion führen.

\section{Schlussfolgerung}

Die Behandlung der Skelettmetastasen hat sich in den letzten Jahren nicht zuletzt durch Fortschritte in der medikamentösen Therapie verändert. Dominierten früher noch rein palliative Therapieverfahren mit der Intention der Schmerzreduktion und Mobilisierbarkeit, sind heute auch durch Verbesserun- 


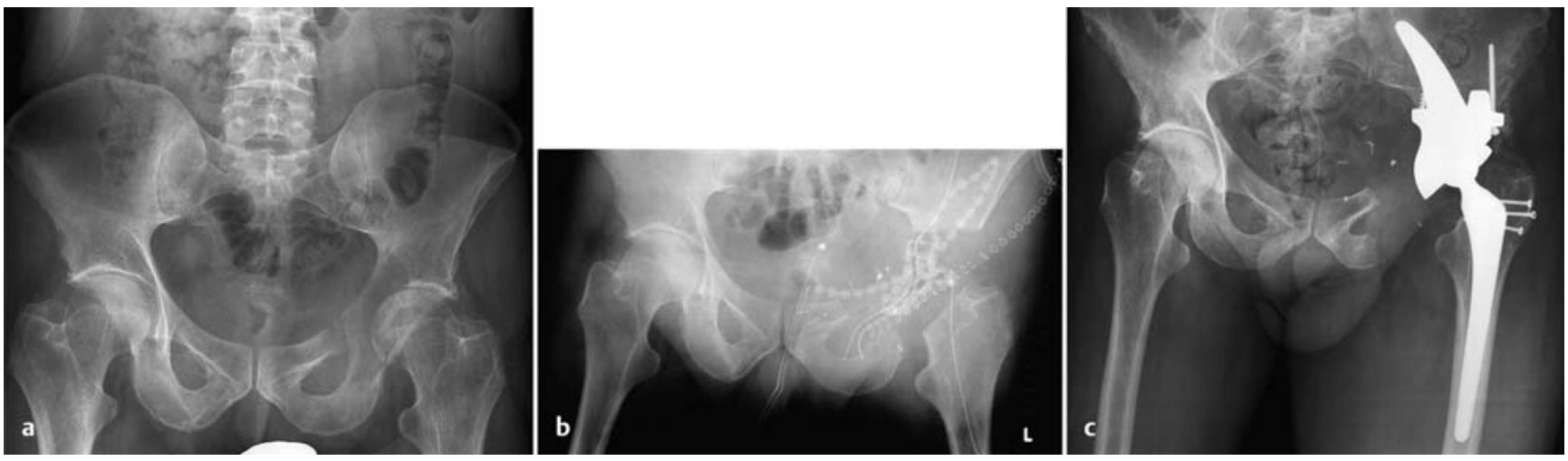

Abb. 5 a bis c a 49-jähriger Patient, 2 Monate nach Nephrektomie bei Nierenzellkarzinom. Es fand sich im Staging eine isolierte azetabuläre Metastase links, sodass die Indikation zur weiten Resektion gestellt wurde. Sekundär sollte dann anhand der Resektion ein Beckenteilersatz gefertigt und in einem 2. Schritt implantiert werden. b Darstellung der periazetabulären Resektion. Es kam zu einer Besiedelung mit Staphylococcus epidermidis, sodass insgesamt 4 Débridements bis zur Keimfreiheit notwendig wurden. c Implantation des Beckenteilersatzes 6 Wochen nach der Resektion. Der Patient entwickelte im Verlauf eine multiple viszerale und knöcherne Metastasierung und erlag 6 Monate nach Implantation seinem Leiden. Die aufwendigere, weitere Resektion mit dem Einbau eines Megaimplantats ist daher nicht kritiklos zu sehen.

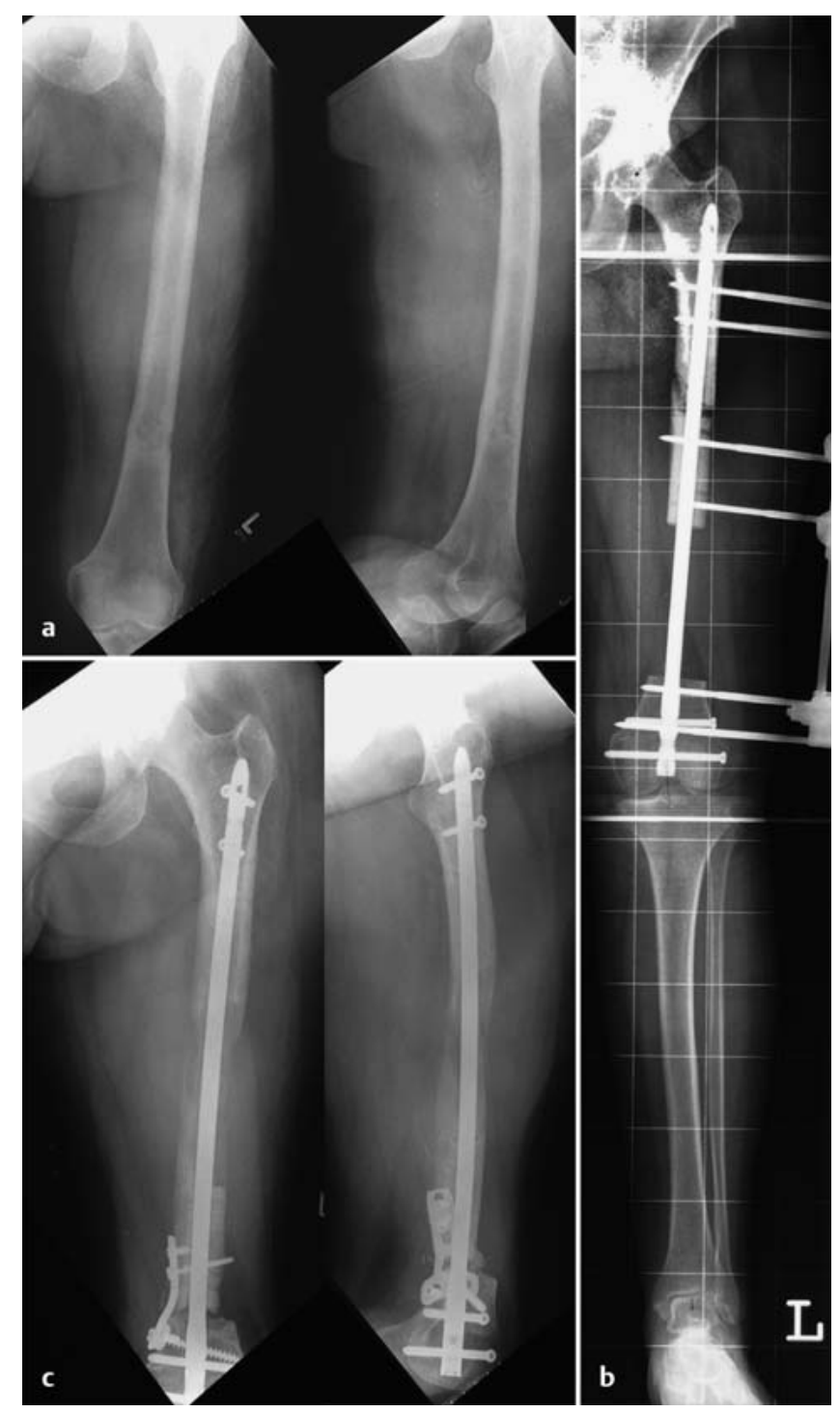

Abb. 6 a bis c a 49-jährige Patientin mit einer isolierten Metastase eines Mammakarzinoms 5 Jahre nach Erstdiagnose und 1 Jahr nach lokaler Strahlentherapie. b Durchführung einer weiten Resektion und Segmenttransport über einen eingebrachten Marknagel. c Knöcherne Konsolidierung nach sekundärer Dockingoperation. Die Patientin ist 8 Jahre nach der weiten Resektion weiterhin tumorfrei mit freiem Gangbild. gen auf dem Gebiet der Tumororthopädie aufwendigere und aggressivere Lokaltherapien verbreitet. In Einzelfällen kann bei isolierten Metastasen gar eine weite Resektion ähnlich der Primärtumorbehandlung indiziert sein. Gute Entscheidungshilfen fehlen, sodass die Art der Behandlung von der Erfahrung des jeweiligen Tumorchirurgen abhängt und an den Patienten und seine Erkrankung adaptiert werden muss.

\section{Literatur}

${ }^{1}$ Bauer HC, Wedin R. Survival after surgery for spinal an extremity metastases. Prognostication in 241 patients. Acta Orthop Scand 1995; 66: $143-146$

2 Tillman RM, Myers GJC, Abudu AT et al. The three-pin modified 'Harrington' procedure for advanced metastatic destruction of the acetabulum. J Bone Joint Surg [Br] 2008; 90: 84-87

${ }^{3}$ Utzschneider S, Weber P, Fottner A et al. Prognoseadaptierte operative Behandlung von Knochenmetastasen. Orthopäde 2009; 38: 308-315

${ }^{4}$ Weber KL, Randall RL, Grossmann S et al. Management of lower-extremity bone metastasis. J Bone Joint Surg [Am] 2006; 88: 11-19

Prof. Dr. med. Thomas Gösling Ltd. Oberarzt der Klinik

Leiter Schwerpunkt Knochen- und

Weichteiltumore

Ulf Brunnemer

Assistenzarzt

Prof. Dr. med. Christian Krettek

Direktor der Klinik

Unfallchirurgische Klinik

Medizinische Hochschule Hannover

Carl-Neuberg-Straße 1

30625 Hannover

goesling.thomas@mh-hannover.de 